TITLE:

\title{
Diverse synchrony of firing reflects diverse cell-assembly coding in the prefrontal cortex.
}

\section{$\operatorname{AUTHOR}(\mathrm{S})$ :}

Sakurai, Yoshio; Nakazono, Tomoaki; Ishino, Seiya; Terada, Satoshi; Yamaguchi, Kenji; Takahashi, Susumu

\section{CITATION:}

Sakurai, Yoshio ... [et al]. Diverse synchrony of firing reflects diverse cell-assembly coding in the prefrontal cortex.. Journal of physiology, Paris 2013, 107(6): 459-470

\section{ISSUE DATE:}

2013-12

URL:

http://hdl.handle.net/2433/179772

\section{RIGHT:}

@ 2013 Elsevier Ltd.; この論文は出版社版でありません。引用の際には 出版社版をご確認ご利用ください。; This is not the published version. Please cite only the published version. 
Diverse synchrony of firing reflects diverse cell-assembly coding in the prefrontal cortex

Yoshio Sakurai ${ }^{\text {a,* }}$, Tomoaki Nakazono ${ }^{\text {a,c }}$, Seiya Ishino ${ }^{\text {a,c }}$, Satoshi Terada ${ }^{\text {a }}$, Kenji Yamaguchi ${ }^{\text {a }}$, Susumu Takahashi ${ }^{\mathrm{b}}$

${ }^{a}$ Department of Psychology, Graduate School of Letters, Kyoto University, Kyoto 6068501, Japan

b Laboratory of Neural Circuitry, Graduate School of Brain Science, Doshisha University, Kizugawa 619-0225, Japan

${ }^{c}$ Japan Society for the Promotion of Science, Tokyo 102-0083, Japan

Corresponding author: Yoshio Sakurai

Postal Address: Department of Psychology, Graduate School of Letters, Kyoto University, Sakyo-ku, Kyoto 606-8501, Japan

Phone and Fax: 81-75-753-2848

E-mail: ysakurai@bun.kyoto-u.ac.jp 


\section{ABSTRACT}

In the present paper, we focus on the coding by cell assemblies in the prefrontal cortex (PFC) and discuss the diversity of the coding, which results in stable and dynamic representations and the processing of various information in that higher brain region. The key activity that reflects cell-assembly coding is the synchrony of the firing of multiple neurons when animals are performing cognitive and memory tasks. First, we introduce some studies that have shown task-related synchrony of neuronal firing in the monkey PFC. These studies have reported fixed and several types of dynamic synchronous firing during working memory, long-term visual memory, and goal selection. The results of these studies have indicated that cell assemblies in the PFC can contribute to both the stability and the dynamics of various types of information. Second, we refer to rat studies and introduce the findings of cellular interactions that contribute to synchrony in working memory, learning-induced changes in synchrony in spatial tasks, and interactions of the PFC and hippocampus in dynamic synchrony. These studies have proposed neuronal mechanisms of cell-assembly coding in the PFC and its critical role in the learning of task demands in problematic situations. Based on the monkey and rat studies, we conclude that cell-assembly coding in the PFC is diverse and has various facets, which allow multipotentiality in the higher brain region. Finally, we discuss the problem of the sizes of cell assembly, how diverse the sizes are in the PFC, and the technical problems in their investigation. We introduce a unique spikesorting method that can detect small and local cell assemblies that consist of closely neighboring neurons. Then, we describe the findings of our study that showed that the monkey PFC has both small and large cell assemblies, which have different roles in information coding in the working brain. 
Keywords: Review, Prefrontal Cortex, Cell Assembly, Firing Synchrony, Crosscorrelation, ICA, Monkey, Rat

\section{Introduction: prefrontal cortex, cell assembly, and firing synchrony}

The prefrontal cortex (PFC) is the most newly developed brain structure, and it is thought to be crucial for many simple fundamental and higher complex functions, including motor execution, cognition, memory, decision making, and thinking (Watanabe, 2007; Fuster, 2008; Passingham and Wise, 2012). Therefore, the functions of the PFC should be considered diverse, nonspecific, and multiple and not defined by a single one. These multiple functions of the PFC might be attributable to the dynamic and diverse coding by cell assemblies, i.e., functional populations of multiple neurons (Hebb, 1949), because cell assembly has been postulated to act as a functional unit that represents information in the working brain.

The original concept of cell assembly is a theoretical notion and it could have value and be substantial when it accounts for experimentally observed phenomena. The experimental observations accounted by the concept of cell assembly are, as Sakurai (1999) suggested, the task-related functional overlapping of individual neurons and the task-dependent dynamics of the functional connectivity of multiple neurons. In particular, the latter phenomenon has often been reported and regarded as the operational definition or indirect evidence of the activity of cell assemblies. Thus, although it might not be a sufficient property of cell assembly, the activity of a cell 
assembly has been inferred to be reflected by the task-dependent synchronized firing of multiple neurons, which indicates the dynamics of functional connectivity among the neurons (Eichenbaum, 1993; Sakurai, 1996a, b; 1999; Riehle, 1997; Engel et al., 2001; Harris, 2005). Therefore, neurons in the PFC are expected to show diverse forms of firing synchrony, which represent diverse and dynamic coding by the cell assemblies, in various behavioral tasks. Actually, several former studies have reported the behaviordependent synchrony (Abeles et al., 1993; Vaadia et al., 1995; Seidemann et al., 1996) and the memory-related synchrony (Funahashi and Inoue, 2000; Constantinidis et al., 2001) of PFC neurons.

The present review focuses on the coding by cell assemblies in the PFC and discusses the diversity of the cell assembly coding, which results in both stable and dynamic representations and various information processes. We introduce the findings of relatively recent studies that have reported several features of firing synchrony, i.e., correlation of firing among multiple neurons, which reflect the diverse and dynamic activities of cell assemblies in the PFC when monkeys or rats perform cognitive and memory tasks. In addition, we discuss the diversity in the sizes, i.e., the ranges in the patterns of activation, of cell assemblies in the PFC. For such a discussion, we introduce new experimental techniques that have been used to detect small and local cell assemblies and introduce our study, which has reported that both small and large cell assemblies function in the PFC.

\section{Diverse synchrony of firing in the monkey PFC}


2.1. Fixed, task-dependent, and event-task-dependent synchrony during workingmemory tasks

The activity of cell assembly, which is reflected by the synchronized firing of neurons, should compensate for the unstable firing of individual neurons and contribute to the stability of the representation of information in the working brain. In addition, it should show dynamic changes that depend on the tasks and the events being processed. The innovative and pioneering study by Funahashi and Inoue (2000) showed such dynamic and task-dependent synchrony in the firing of monkey PFC neurons. Their crosscorrelation analyses showed significant functional interactions among PFC neurons having various task-related activities during a working-memory task. Their results surely suggested that PFC neurons interact with each other to manipulate and integrate various information during the processing of working memory.

We also have analyzed synchronous firing of neurons in the PFC of monkeys during processes of different working memory (Sakurai and Takahashi, 2006). The monkeys successively performed 2 tasks in which working memory for either stimulus duration or color was required. During the performance of the tasks, we recorded the activities of multiple neurons in the dorsolateral PFC. The method of multineuronal recording used arrays of tetrodes that were attached to microdrives with a distance between the nearest neighbors of $500 \mu \mathrm{m}$ (McNaughton et al., 1989; Sakurai, 1996a; 2002; Sakurai et al., 2004), and we recorded the activities of the same multiple neurons for more than $2 \mathrm{~h}$ while the monkeys were performing the 2 different tasks. The recorded neuronal activities were analyzed by a unique spike-sorting method (Takahashi et al., 2003a, b) 
that could separate spikes from individual neighboring neurons and detect their precise synchrony, even when their waveforms overlapped with each other.

A total of $48 \%$ of 1,405 neuronal pairs that were recorded from the PFC showed synchronous firing, revealed by cross-correlation analysis, during the performance of the tasks. Almost half of such neuronal pairs showed fixed synchrony, and they constantly fired together in both of the tasks. However, some neuronal pairs showed task-dependent synchrony, and firing appeared in only one of the tasks (Fig. 1). Moreover, the other neuronal pairs showed event-task-dependent synchrony. Firing appeared during stimulus presentation or retention periods in the tasks, and the periods that showed synchrony varied between the tasks. These results indicated that the cell assemblies in the monkey PFC were diverse and had different properties of effective connectivity (Aertsen et al., 1989) that were revealed by firing synchrony. One form of cell assembly consisted of neurons that showed robust synchronous firing across different types of tasks and events. This form contributed to the stable representation of information, and the neuronal pairs that showed fixed synchrony were members of such a cell assembly. The other forms of cell assembly consisted of neurons that also fired synchronously with each other but with a synchrony that dynamically changed depending on the types of tasks and events to be processed. The neuronal pairs showing task-dependent synchrony and event-task-dependent synchrony were members of such dynamic cell assemblies.

In Sakurai and Takahashi (2006), we have reported that the task-dependent synchrony of the neuronal pairs was not dominant in either of the 2 working-memory tasks that required the monkeys to retain and process temporal information (presentation periods) or stimulus information (stimulus colors). This indicated that the cell assemblies in the 
PFC are not biased to the processing of one specific type of information. In addition, there was no event-task-dependent synchrony that appeared in any of the same event periods in both tasks. It appeared in different event periods between the tasks or in 1 or 2 event periods in one of the tasks. This meant that the event-task-dependent synchrony depended not on the physical attributes of the events, such as stimulus presentation or retention intervals, which were common for both tasks, but on information attributes of the events, such as encoding, retention, and/or the comparison of valid information, which were different between the tasks. Therefore, the monkey PFC has diverse cell assemblies, as reflected by the event-task-dependent synchrony, that dynamically represent different information in different tasks.

Although approximately half of the neuronal pairs with firing synchrony showed the dynamic task-dependent or event-task-dependent synchrony that reflects the diversity of cell-assembly coding, the remaining half showed fixed synchrony. Almost all of the neuronal pairs that showed fixed synchrony were recorded from 1 tetrode, and they had sharp peaks of correlation like the peaks that appeared in task-dependent synchrony. These constantly coactive neighboring neurons might have been parts of cell assemblies, together with neurons that have dynamic functional connectivity and that provide functional stability and compensation for unstable firing and the destruction of individual neurons.

2.2. Dynamic synchrony that depends on behavioral performance and stimuli in a visual-memory task 
The PFC has often been suggested to play important roles not only in working memory, but also in organizing higher executive functions in long-term memory, especially in visual memory (Miller, 2000; Fuster, 2008). PFC neurons have been predicted to synchronize their spiking when visual information is entered and processed to construct visual images and memory because of the convergence of multiple visual pathways onto the PFC. Furthermore, spike synchrony should intensify more when more neural plasticity is required to process the memory. Pipa and Munk (2011) have tested whether spike synchrony that was detected by their multisite prefrontal recordings was dependent on behavioral performance in a visual-memory task and on the to-be-remembered stimuli in the task.

They trained monkeys to perform a short-term visual-memory task and simultaneously recorded multineuronal activity from the ventral PFC with electrodes that were arranged in a square-shaped $4 \times 4$ grid with a distance between the nearest neighbors of $500 \mu \mathrm{m}$. They referred to each incidence of synchronized firing within a 3ms time window as a joint-spike event (JSE) and to the identity of JSEs as a joint-spike pattern (JS-pattern). They generated surrogate data that were derived from the original data by jittering each individual spike train within a 15-ms time window. JS-patterns were considered significant if there were significantly more JSEs in the original data than in the surrogate data set. JS-patterns were grouped based on their complexity (c), which was determined by the number of electrode sites that participated in a synchronous event, and JS-patterns with complexities that ranged from 2 to 8 were analyzed. They slid time windows in order to determine whether JS-patterns were modulated dynamically in the time course of information processing for the memory 
task and whether they were dependent on task performance, such as the correct or incorrect responses and/or the to-be-remembered stimuli that were presented in the task.

Figure 2 shows that the differences (z-scores) in the significant JS-pattern frequencies between the correct and incorrect trials for each complexity and each sliding time window $(400 \mathrm{~ms})$ that was modulated as a function of the trial time course (time after sample onset). This indicated that JS-patterns dynamically changed their frequencies and complexities depending on the behavioral performances in the task. The stimulusspecific modulation of JS-patterns was also observed during presentation of the sample and test stimuli. These results indicated that spike synchrony with high temporal precision across electrode sites that were separated by $500 \mu \mathrm{m}$ was detected in the ventral PFC and that the frequency of synchrony was modulated depending on the behavioral performance and the specific stimuli that were presented. In particular, during the delay period, larger groups of up to 7 sites showed performance-dependent modulation of their spike synchronization.

The finding that spike synchrony with a 3-ms temporal precision across several electrode sites was dynamically modulated depending on the behavioral performance could reflect the dynamic activity of large cell assemblies that underlie the higher temporal organization of executive processes. The precise temporal synchrony of such a large neural population could play a critical role in the higher information processing of memory. Moreover, the finding that synchronous spiking was also modulated by specific visual stimuli suggested that the cell assemblies that were reflected by the spike synchrony were also involved in the sensory mechanisms for visual information processing in the PFC. 
The approach by Pipa and Munk (2011) was prominent and successful. They, however, had a methodological limitation. They analyzed firing synchrony among distant neurons, but they could not analyze synchronous spikes that occurred among neighboring neurons at individual electrode sites because they recorded spike signals with single-ended microelectrodes. Such a limitation could be overcome by recording with specific multielectrodes and spike-sorting methods. We discuss this issue in the last section of this review.

\subsection{Various types of synchrony and their dynamics in goal-selection tasks}

The dynamic synchrony of monkey PFC neurons has been reported in other higherorder information processes, such as those involving goal selection. In a series of experiments, Wise and his colleagues have investigated the activity of PFC neurons in monkeys processing information for goal selection. They employed an abstract strategy task (Genovesio et al., 2005), in which the monkeys were required to select a future (next) goal on the basis of memory of the previous goal. A hallmark of this behavioral paradigm is that signals for the previous goals and future goals are processed during different delay periods. Therefore, the experimenters could distinguish the information processes for the two goals and observe the neuronal dynamics for goal selection. They recorded PFC neuronal activity during the task and examined which neurons responded to the previous goals, future goals, or both goals. They found that PFC neurons encoded both goals at the level of population activity. However, interestingly, individual neurons typically encoded either previous or future goals, but not both goals (Genovesio et al., 2006). They called the population of PFC neurons that encoded previous goals P cells 
and the population that encoded future goals F cells. They concluded that the PFC has separate neural populations that encode these two types of goals.

Their next question was how $\mathrm{P}$ cells and F cells interact with each other for decision making in selecting a goal because the information about previous goals is indispensable in choosing a next goal in the task. In order to answer this question, they evaluated the correlated activities in each pair of neurons (Tsujimoto et al., 2008). They recorded the activity of PFC neurons with movable microelectrodes and identified the cell types as $\mathrm{P}$ cells or F cells. Then, they computed joint perievent time histograms (JPETHs), which are two-dimensional histograms that display the correlated activity of each pair of simultaneously recorded neurons. With this analysis, they assessed the relationships between the time courses of the synchronized firings of the neuron pairs and the properties that were encoded by each neuron ( $\mathrm{P}$ cell or $\mathrm{F}$ cell). First, they revealed that there were different amounts of neuronal proportions that showed significant positive correlations due to the combinations of cell types. The percentages of F-P and F-F pairs, but not P-P pairs, were significantly higher compared to those of randomly selected control pairs. Furthermore, the time courses of the correlation coefficients of F-P pairs and F-F pairs were different (Fig. 3). Although both F-P pairs and F-F pairs showed increased correlation coefficients during the cue presentation periods, the correlation coefficients of F-P pairs continued to increase, even after goal achievement. On the other hand, during the fixation periods prior to the cue presentation periods, only F-F pairs showed increases in their correlation coefficients. Considering these JPETH time courses, they assumed that the correlated activities of F-F pairs contributed to the selection and active maintenance of future goals because the 
decreases in the correlations after goal achievement were consistent with prospective coding.

The common feature during cue presentation suggested that F-P pairs also contributed to the selection and maintenance of goals. However, the continued enhancement of the correlation after the cue went out indicated another role of F-P pairs. They suggested that F-P pairs had the ability to remember the goal that was chosen not only in the previous trials, but also in the current trials. Because their task had 3 possible goals, monkeys should remember, at most, the 2 choices that were selected in the 2 recent previous trials in order to suppress repetitive selection of prior choices and select the correct (remaining) goal in the next trial. This role could be called monitoring (Tsujimoto et al., 2008).

From the point of view of the neuronal mechanisms of synchronized firing, we can presume that the functions of the correlations of F-F pairs are to discharge downstream target neurons efficiently (König et al., 1996) and to convey shared spatial information robustly. This could be called reader mechanism (Buzsaki, 2010). In F-F pairs, downstream reader neurons read out the spatial information of a future goal. The function of reader neurons in F-P pairs is to integrate the information of the 2 goals into the goal in the next trial. These studies by Wise and colleagues have suggested that the functions of the synchronized firing of PFC neurons are not uniform but are dynamic and diverse.

Sakamoto et al. (2008) have suggested another role of the spike synchrony in PFC. They analyzed the time courses of the synchrony of PFC neuronal pairs that were recorded from monkeys that were performing a path-planning task. In their task, the monkeys are required to plan an immediate goal in order to attain a final goal. The 
firing synchrony of PFC neurons during the task was maximized during the transitional periods between the immediate goals and the final goals. They assessed that such enhanced synchrony represented the process of the facilitation of the dynamic changes in the neural circuits from one steady state to another. This assumption is consistent with a theoretical study (Aoki and Aoyagi, 2007) that has proposed that the role of spike synchrony is to not only represent and process information but also to transfer an activity state of neuronal networks to a different state in order to realize the dynamic coding of information.

Consequently, the studies that were described above suggested that the cell assemblies in the monkey PFC have diverse functions. As monkey PFC neurons have various roles, it might not be strange that the combination of these various types of neurons make the functions of their synchronized firing more complex and diverse.

\section{Diverse synchrony of firing in the rat PFC}

\subsection{Dynamic synchrony between pyramidal neurons and interneurons during a working-memory task}

The PFC has also been widely studied in rats. It has often been suggested that rat PFC, as well as monkey PFC (see section 2.1 of this review), has the appropriate properties for working memory (Funahashi, 2006) in that short-term plasticity induces both the facilitation and the depression of synapses (Wang et al., 2006). This property indicates that the functional connectivity in the PFC can vary in fine time scales, which can be represented as the dynamics of synchrony among neurons. Although a great deal of in 
vitro and computational research has supported this hypothesis (Gupta et al., 2000; Hempel et al., 2000; Markram et al., 2004; Sussilo et al., 2006; Wang et al., 2006; Mongillo et al., 2008), empirical evidence that reflects the mechanisms of the neuronal connections during working-memory-dependent behavior has been scarce.

An important experiment that examined this problem was conducted by Fujisawa et al. (2008), and they focused on the sharp peaks in cross-correlograms between pyramidal neurons and interneurons with ms time lags that were consistent with monosynaptic delays. They examined the firing patterns and the temporal relationships of the activities of medial PFC neurons during a working-memory task, which was an odor-based delayed-match-to-sample T-maze task. The rats were required to choose either a right or left arm according to an odor cue. Specifically, if the cue was a cheese odor, the rats were given reward when they ran to the end of the right arm. If the cue was chocolate, running to the end of the left arm was correct and rewarded. First, the authors found that the sequential activities of widely distributed PFC neurons were reliably different between the trajectories that corresponded to the rat's choice of arms and that the individual neurons were differently active only for a short duration (Fig. 4A). Importantly, a number of monosynaptic pairs between the pyramidal neurons and interneurons of the PFC dynamically varied their peaks in the cross-correlograms across various phases of the task beyond the statistical accounting for the effects of covarying the firing rates of the neurons (Fig. 4B). This indicated that the functional connectivity between the PFC neurons was dependent on the behavioral performance in the task. Moreover, like that observed in previous in vitro observations (Gupta et al., 2000; Markram et al., 2004), they statistically indicated that such a monosynaptic interaction can possess either depressing or facilitating properties and that the coincident firing of 
plural neurons leads to the effective interactions between neuron pairs. These findings suggested that functional connectivity can vary dynamically according to task demands which work out the internally generated representation and require goal representation and motor sequences. Such task demands are embedded in the sequential changing of the activity of PFC neuronal populations.

Thus, Fujisawa et al. (2008) have examined the interactions in the local neocortical networks of neurons during working-memory-dependent behavior, and their findings supported the hypothesis of phase sequences of cell assemblies (Hebb, 1949). Notably, they uncovered a neuronal mechanism of cell assembly and assumed that the functional connectivity in it was a monosynaptic interaction between pyramidal neurons and interneurons. This finding was consistent with those of previous studies that haveobserved variance in the short-term synchrony between neuronal pairs as a function of behavioral performance and learning (Constantinidis et al., 2002; Baeg et al., 2007), in which they assumed that putative monosynaptic interactions between two PFC neurons were mostly negative and likely involved an inhibitory interneuron (Constantinidis et al., 2002). Consequently, PFC could have diverse patterns of synchronized firing among different and same types of neurons, which allow the PFC to be involved in various higher cognitive functions, such as working memory.

\subsection{Learning-induced changes of firing synchrony in spatial-navigation tasks}

The hypothesis of cell assembly (Hebb, 1949) postulates that cell assemblies are formed and modulated by learning. Baeg et al. (2007) have investigated whether the functional connectivity of PFC neurons constructing cell assemblies, which is reflected 
by synchronous firing, changes in parallel with behavioral learning, whether such functional connectivity changes repeatedly during different learning tasks, and whether the changes of functional connectivity are maintained stably for a long time in supporting long-term memory.

They used a 8-shaped maze for spatial-navigation tasks and a long-term ensemblerecording technique to record multineuronal activity during both learning- and memoryretention periods across multiple days. They trained rats to acquire 2 types of behavioral rules in parallel. In phase $\mathrm{I}$, the rats were trained to run toward a right or left spatial location alternately (task 1). In phase II, which followed the training of task 1 for 25 trials, running toward the left side was blocked by a wall that was inserted in the maze. Then, they were trained to turn right repeatedly (task 2). After phase II was conducted for 14 days, task 2 was trained continuously for 1-31 days (overtraining phase).

The results of their analysis showed that, during the course of learning, both the enhancement and reduction of firing synchrony occurred in the same range (Fig. 5A and B). Synchrony changes occurred more frequently at phase I when the task performance progressively increased, but such increments of firing synchrony were not observed in the latter phases when the learning reached a learning asymptote (Fig. 5C and D). As the variations and means of firing rates or bursts were not significantly different between phases I and II, these findings supported the notion that functional connectivity changed in parallel with behavioral learning. In addition, the altered functional connectivity remained stable because the firing synchrony in the overtraining phase was comparable with that in the latter parts of phase I and II when the animals were performing the task at an asymptotic level. This suggested that the learning-induced changes of firing synchrony encoded long-term memory in the task. 
In order to investigate the differences in neural activity between different learning tasks, they compared the neural patterns between task 1 and 2 when the rats were running or resting at the same parts of the maze in the overtraining phase. The results showed that, although the animals ran in the same maze, the patterns of neural activity, or the increased or decreased firing rates, were quite different between the 2 tasks. The variations and means in the firing rates between task 1 and 2 were not different significantly. This indicated that the different neuronal activity patterns in the PFC that were observed during the 2 tasks represented 2 different memories. However, even as the individual neural activities were dependent on the tasks, the firing synchronies of the neuron pairs were stable, and this was independent of the tasks. These results suggested that the functional connectivities among the neurons remained similar across the 2 tasks in the overtraining phase, regardless of the changes in individual neural activities.

In conclusion, Baeg et al. (2007) have shown that the changes in the firing synchrony of PFC neurons are greater during the early stages of learning but become progressively smaller as learning reaches an asymptote. Once functional connections are formed, the connections are maintained stably, independent of the task rules, such as the content of memories, or the individual neuronal activity patterns. Baeg and his colleagues have noted that these learning-induced changes in firing synchrony provide crucial evidence for the notion that memories are encoded by the altering patterns of synaptic strengths among coactivated neurons.

\subsection{Interactions of the PFC and hippocampus in dynamic synchrony}


In Baeg et al. (2007), the authors have claimed that the changes in the synchronized firing in the PFC with learning were not affected by changes in the afferent activity from the hippocampus. They have shown that changes in the functional connectivity in the PFC were not dependent on the animal's positions, even though individual neuronal activities in the hippocampus had place specificity. Recently, however, some studies have suggested the possibility that the hippocampus can affect cell assembly dynamics that are reflected by firing synchrony in the PFC. Especially, a local field potential (LFP) that is recorded from the hippocampus represents such an effect on the firing synchrony in the PFC. Jones and Wilson (2005) have analyzed the interactions between theta oscillations of LFPs in the hippocampus and neuronal firings in the PFC. They have shown that the timing of PFC neuronal firing, which could contribute to firing synchrony, was locked with the phases of the theta cycles of hippocampal LFPs when the rats were performing a working-memory task.

Benchenane et al. (2010) have suggested that the activities of PFC cell assemblies are related to interactions between LFPs of the PFC and the hippocampus. They recorded PFC spike activity and LFPs in the PFC and the hippocampal CA1 simultaneously from rats learning multiple rules in Y maze tasks. First, they measured the coherence between LFPs of both areas, and they observed a strong coherence in the theta band (5-10 Hz). This coherence had peaks at the fork (the choice point) of the Y maze. This increase of coherence was significantly greater after the acquisition of a new rule than a former rule. Next, they investigated PFC neuronal activity and applied a principal component analysis (PCA) to the matrix of binned simultaneously recorded spike trains. This method was able to extract neuronal groups that were reliably coactive. They considered these coactive groups of neurons cell assemblies. The activity of these assemblies 
increased in the periods when the hippocampus-PFC theta coherence was increased, and it peaked when the rats were at the choice point of the maze. Moreover, their activities were modulated by the theta oscillations of both areas and increased at the trough of the theta rhythms. Those observations suggested that the activities of PFC cell assemblies were dependent on hippocampal theta oscillations and that this interaction played an important role in learning. The study of Benchenane et al. (2010) also has implied the possibility of the interplay of cell assemblies between the PFC and the hippocampus. As the cell assemblies in the hippocampal CA1 are active at the troughs of its theta oscillations (Harris et al., 2003), the activities of the cell assemblies of the PFC and the hippocampus might be commonly related to theta oscillations.

It has often been suggested that hippocampal cell assemblies contribute to learning and memory formation. Recently, Nakazono et al. (2012) have reported that the synchronized firings of hippocampal neurons were changed by learning a rule in a nonspatial task. Furthermore, Kucewicz et al. (2012) have analyzed the activity changes of cell assemblies in the PFC and the hippocampal CA1 during learning. They recorded multineuronal activities from the PFC and the hippocampus simultaneously in rats that were performing the working-memory task, a delayed-nonmatch-to-position task. They detected hippocampus-PFC neuronal assemblies with the PCA and showed that the activities had relationships with the sample presentation and/or delay periods of the task. Considering the findings of these studies, it is possible that the interactions between not only the hippocampal LFPs and the PFC spike activities, but also the hippocampus-PFC cell assemblies, contribute to learning.

Recent technological advances have made it possible to record from larger neuronal populations. New PCA methods (Peyrache et al., 2009; Lopes-dos-Santos et al., 2011) 
are suitable for detecting large cell assemblies that are constructed by a number of neurons. However, classical methods, such as cross-correlation analyses, have merit in the speculation of the functional connectivities between neurons. A combination of the new methods of PCA and the classical methods may be ideal in analyzing cell assembly dynamics. The next section of this review further discusses the technical problems in the detection of the real features of cell assembly dynamics.

\section{Diverse sizes of cell assemblies in the PFC}

\subsection{Problems in detecting a small and local cell assembly}

So far in the review, we have introduced and discussed the diversity of the functional features of cell assemblies in the PFC, which are reflected by diversity in the synchrony of firing in that area. This last section focuses on the problem of the sizes of cell assemblies (Sakurai and Takahashi, 2008) and discusses how diverse the sizes of cell assemblies are in the PFC.

Because a cell assembly is a functional population and not a structural population of neurons, its size does not reflect the structural size of a neuronal population but instead reflects the range of the patterns of activation that are caused by the diffuse populations of neurons that are capable of acting briefly as a closed system (Hebb, 1949). The large or small size of a cell assembly, therefore, is based on the functional connections of the neurons, and it should vary and depend on the brain region and the type of information being processed in that region (Eichenbaum, 1993; Sakurai, 1996b). It can be predicted that, in sensory and motor cortices where segregated information is efficiently processed, 
in the hippocampus where information is processed for integration, and in the PFC where various information is expected to be dynamically processed, the coding by the cell assemblies may differ significantly with each other. Eichenbaum (1993) and Sakurai (1996b) have suggested a hypothetical framework that organizes several features of cell-assembly coding. The cell-assembly coding in the PFC, which has the most diverse and dynamic functional roles, is expected to possess both features of the sensory/motor cortices and the hippocampus and show synchronized firing among small numbers of neurons in a narrow restricted area and among many neurons in a broad area. Consequently, the size of cell assemblies in the PFC depends on the various types of information that are being represented, and they can be both local and broad.

As the size of cell assemblies varies, the PFC should show firing synchrony that depends not only on tasks and events, but also on the distances among the neurons. However, it is difficult for this assumption to be fully confirmed due to a technical problem. Synchrony among distant neurons or groups of neurons that form a large assembly or separated assemblies can be easily detected with distantly located electrodes (Deadwyler et al., 1996; Buzsaki, 2004; Pipa and Munk, 2011), and in particular, it can be detected by observing the averaged potential of a neuronal population, e.g., optical imaging, LFP, and electroencephalography (Arieli et al., 1996; Kahana, 2006). In contrast, it is difficult to detect activities of closely neighboring single neurons that form a local cell assembly because it is problematic to separate the extracellular activities of neighboring neurons and detect the firing synchrony among them.

The main reason for this is the problem of spike sorting (Lewicki, 1998). With ordinary spike-sorting techniques, different spike amplitudes and waveforms are used to 
classify single neuronal activities, and such techniques are commonly used when multineuronal activities are recorded by a specific electrode with multiple tips, such as tetrodes. However, the action potentials of individual neurons sometimes have varying shapes (Fee et al., 1996), and, at the same time, different neurons sometimes generate action potentials that have very similar waveforms. The latter happens when the neurons are similar in morphology and about equally distant from the electrode tips. It is also well known that some extracellular recordings commonly show characteristic bursts called complex spikes. A stereotyped feature of these bursts is a progressive variation in spike shape during the burst. These problems limit the isolation accuracy of spike sorting by template-matching methods, which are based on the assumption that spike shapes that are generated from the same neuron are similar.

In order to avoid the problems of template matching, a technique called cluster cutting is often used (e.g., Wilson and McNaughton, 1993). In this approach, the user defines a boundary for a particular set of features. Multiple recordings of the same neuron from different physical locations of the electrode tips allow additional information to be used for more accurate cluster cutting. It is apparent, however, that the positioning of boundaries for optimal classification is sometimes very difficult when the clusters are not distinct (Takahashi et al., 2003a, b). In order to avoid such difficulty in the positioning of the boundaries, automatic clustering by a PCA (Abeles and Goldstein, 1977) can be performed with multiple recording channels in order to obtain useful features for cluster cutting. Although such cluster cutting by PCA can be reliable for some data sets in which spike waveforms are nearly stationary, there are several factors that prevent one from making a simple (Gaussian) assumption of stationary spike waveforms (Takahashi et al., 2003b), and individual neurons often generate 
nonstationary waveforms, particularly when they fire in the form of complex bursts. These make it difficult to define clusters of individual neuronal activities.

Another serious problem with detecting the synchrony among neighboring neurons is spike overlapping (Gray et al., 1995; Lewicki, 1998). When multiple neighboring neurons fire simultaneously, spike waveforms generated from the neurons overlap on the near electrode. Ordinary spike-sorting methods, including cluster cutting, detect the overlapping spikes as outliers, and there is no means of determining whether some closely neighboring neurons generate simultaneous action potentials. In order to overcome this problem, Lewicki (1994) has developed a computationally efficient overlap decomposition algorithm. This decomposition procedure, however, has a limitation because it assumes that the spike clusters are spherical, which is equivalent to assuming fixed stationary spike waveforms. These problems make it difficult to sort the activities from closely neighboring neurons and detect the precise synchrony among the neighboring neurons that form a local cell assembly.

\subsection{Innovating spike sorting with independent component analysis (ICA)}

We have solved the problems of spike sorting, that is, that of nonstationary waveforms and spike-overlapping problems, and have proposed a unique method that combines independent component analysis (ICA) (Comon, 1994) and an ordinary sorting method (k-means clustering) (Takahashi et al., 2003a, b). This method, which was briefly introduced in section 2.1 of this review, can sort the activities of closely neighboring neurons in behaving animals. Although ICA alone can solve the problems, it has a serious limitation that the number of separated single neurons must be less than 
the number of electrode tips, meaning that a tetrode (4 electrode-tips), for example, can detect less than 4 neurons from 1 recording site. Therefore, we have combined ICA and the efficiency of the ordinary spike-sorting technique to solve both the nonstationary and the spike-overlapping problems with no limitations on the number of single neurons to be separated (Takahashi et al., 2003a, b). A chart showing the summary of the steps in the algorithm is shown in Fig. 6. This procedure can be extended to an analysis of recordings that have numbers of simultaneously acquired channels that are larger than that of the tetrode.

The results of the study by Sakurai and Takahashi (2006), introduced in section 2.1 in this review, have confirmed that our spike-sorting method was able to separate single neurons from the multineuronal activities that were recorded with tetrodes, even when they had overlapping spikes and attenuating waveforms that were caused by spike bursts. Figure 7 shows a detailed example of raw data, and it includes plots of raw signals from a single tetrode in both slow (Fig. 7A) and fast (Fig. 7B) time scales. The box in the dotted lines in Fig. 7B is a period when spikes from 2 of the neurons overlapped. Fig. 7C shows the overlapped and separated waveforms of the spikes from the 2 neurons. This example indicates that the present method can separate single neuronal spikes, even when they sometimes overlap. Figure 8 shows an example of raw data from a single neuron that was separated with the present method with the tetrode data shown in Fig. 7. It includes a scatter plot of spike clusters (Fig. 8A), averaged waveforms that were recorded from each of the 4 electrode-tips (chs.) of the tetrode (Fig. 8B), and the autocorrelogram of the firing of the neuron (Fig. 8C). The peaks on both sides of the zero in the autocorrelogram (Fig. 8C) indicate that the neuron generates spike bursts, and the widely distributed points in the cluster (Fig. 8A) indicate that the 
spike bursts caused waveform attenuation. Even at the 2 points of the spikes that were most distant from each other ( $a$ and $b$ ), which imply large differences in their waveforms, the serial patterns of the waveforms on the 4 tips of the tetrode are similar (Fig. 8B), and the correlation coefficient between the waveform patterns of the plots for $a$ and $b$ is 0.75 . Besides such waveform consistency, the clear refractory period shown in Fig. 8C indicates that the cluster is from a single neuron. This example strongly indicated that the present method can separate single neurons, even when they generate spike bursts that cause waveform attenuations.

\subsection{The monkey PFC has both local and broad cell assemblies}

In Sakurai and Takahashi (2006), we divided all neuronal pairs that show synchrony of firing into 2 groups, with one group containing the neuronal pairs recorded from 1 tetrode and the other group containing the neuronal pairs recorded from 2 adjacent tetrodes that were $500 \mu \mathrm{m}$ apart. Figure 9 shows a summary of the proportions of the neuronal pairs that showed fixed, task-dependent, and event-task-dependent synchrony, which are described in section 2.1 in this review, that were recorded from 1 tetrode (Fig. 9A) and from 2 tetrodes (Fig. 9B). It is apparent that most of the neuron pairs in all of the types of synchrony were recorded from 1 tetrode. The event-task-dependent synchrony, however, had significantly fewer neuronal pairs that were recorded from 1 tetrode (Fig. 9A) and more neuronal pairs that were recorded from 2 tetrodes (Fig. 9B) than the other types of synchrony.

We also classified the synchrony peaks as either sharp or broad. The former was defined as a narrow significant peak ( $<10$-ms width at the level of the confidence limit), 
and the latter was defined as a wide significant peak $(\geq 10$-ms width at the level of the confidence limit) in the correlograms. We then divided all of the neuronal pairs that showed synchrony into 2 groups, namely, the sharp-synchrony and broad-synchrony groups. Fig. 9 illustrates the proportions of neuronal pairs with sharp and broad synchrony in the neuronal pairs that were recorded from 1 or 2 tetrodes. In all types of synchrony, it is clear that most of the neuron pairs that showed sharp synchrony were recorded from 1 tetrode (Fig. 9A). However, most of the neuronal pairs that showed broad synchrony were recorded from 2 tetrodes (Fig. 9B).

The above findings suggested that the neuronal pairs that showed task-dependent synchrony and event-task-dependent synchrony were members of local and broad cell assemblies, respectively. As almost all of the neighboring neurons that were recorded from 1 tetrode showed sharp peaks of synchrony (Fig. 9), the spike timings among the neurons comprising a small cell assembly were very constant with little variation. From the simultaneous intra- and extracellular recordings (Henze et al., 2000), the diameter of the recording area of 1 tetrode was about $100 \mu \mathrm{m}$. This indicated that the neighboring neurons in an area of about $100 \mu \mathrm{m}$ tended to fire synchronously to form a local and small cell assembly in the PFC. Although not like many of the neuron pairs that were recorded from 1 tetrode, a small number of neuronal pairs that were recorded from 2 tetrodes that were $500 \mu \mathrm{m}$ apart showed firing synchrony, and event-task-dependent synchrony was observed more frequently in the distant neuronal pairs than the other types of synchrony (Fig. 9). This suggested that some broad and large cell assemblies that were reflected by the event-task-dependent synchrony might have been formed by more distant neurons. The long-distance and multisynaptic connections among neurons forming a broad cell assembly or separate cell assemblies might make it possible to 
dynamically change the effective connectivity in a time period that is on the long order during the events, such as stimulus presentation and retention periods, in the tasks. The larger variations of spike timings in the broad synchronies between distant neurons might contribute to the dynamic changes in effective connectivity.

The results of our study, in consequence, suggest that the PFC has both local (small) and broad (large) cell assemblies that fire synchronously with each other and that the synchrony dynamically changes depending on the type of information, such as tasks and/or events, to be represented and processed.

\section{Toward direct evidence of the diverse coding of cell assemblies in the PFC}

The present review focused on the diversity of the coding of cell assemblies in the PFC and introduced and discussed recent studies that have reported diverse features of firing synchrony in multiple PFC neurons and the new techniques that are used to more precisely detect such firing synchrony. However, as described in section 1, the concept of cell assembly was originally a theoretical notion. The several forms of taskdependent firing synchrony among neurons that were introduced in the present review were indirect evidence for the cell assembly's activation and do not constitute direct evidence of the existence of cell assemblies. Alternative theoretical notions other than the concept of cell assembly could account for the task-dependent firing synchrony. Therefore, we should broaden our outlook with more advanced experimental techniques and theoretical approaches that could reveal direct evidence of cell assembly coding.

A promising experimental technique that could reveal the real features of the activity of cell assemblies might be the real-time imaging of a large number of neurons in the 
working brain (e.g., Ikegaya et al., 2005). This technique, however, detects neuronal activities only from the surface or shallow structures of the brain. Because of this limitation in the experimental technique, synergistic views involving more sophisticated theoretical and experimental neuroscience that are compatible with and account for the experimental data are also needed (e.g., Ainsworth et al., 2012). Whatever theoretical frameworks are employed and whatever techniques are used to observe the activity of cell assemblies in future studies, it is most important to define the types of information processes in the working brain. The reason is that the cell assemblies must be diverse and dynamic, especially in the PFC, and their features must be task-dependent and vary according to the types of information and the processing. Thus, the use of behaving animals in behavioral tasks is essential, and psychological consideration of the tasks should not be neglected.

\section{Acknowledgements}

This work was supported by the Core Research for Evolution Science and Technology (CREST) program and JSPS KAKENHI grant Nos. 20243035 and 24243069 (Y.S.) and 24300148 and 25560435 (S.Takahashi).

\section{References}

Abeles, M., Bergman, H., Margalit, E., Vaadia, E., 1993. Spatiotemporal firing patterns in the frontal cortex of behaving monkeys. Journal of Neurophysiology 70, 16291638. 
Abeles, M., Goldstein. M., 1977. Multispike train analysis. Proceedings of the IEEE 65, $762-773$.

Aertsen, A.M., Gerstein, G.L., Habib, M.K., Palm, G., 1989. Dynamics of neuronal firing correlation: modulation of "effective connectivity". Journal of Neurophysiology 61, 900-917.

Ainsworth, M., Lee, S., Cunningham, M. O., Traub, R. D., Kopell, N. J., Whittington, M. A., 2012. Rates and rhyshms: A synergistic view of frequency and temporal coding in neuronal networks. Neuron 75, 572-583.

Aoki, T., Aoyagi, T., 2007. Synchrony-induced switching behavior of spike pattern attractors created by spike-timing-dependent plasticity. Neural Computation 19, $2720-38$

Arieli, A., Sterkin, A., Grinvald, A., Aertsen, A., 1996. Dynamics of ongoing activity: explanation of the large variability in evoked cortical responses. Science 273, 18681871.

Baeg, E.H, Kim, Y.B., Ghim, J.W., Kim, J.J., Jung, M.W., 2007. Learning-induced enduring changes in functional connectivity among prefrontal cortical neurons. Journal of Neuroscience 27, 909-918.

Benchenane, K., Peyrache, A., Khamassi, M., Tierney, P.L., Gioanni, Y., Battaglia, F.P., Wiener, S.I., 2010. Coherent theta oscillations and reorganization of spike timing in the hippocampal- prefrontal network upon learning. Neuron 66, 921-36.

Buzsaki, G., 2004. Large-scale recording of neuronal ensembles. Nature Neuroscience 7, 446-451.

Buzsáki, G., 2010. Neural syntax: cell assemblies, synapsembles, and readers. Neuron $68,362-385$. 
Comon, P., 1994. Independent component analysis, a new concept? Signal Processing $36,287-314$.

Constantinidis, C., Franowicz, M.N., Goldman-Rakic. P.S., 2001. Coding specificity in cortical microcircuits: a multiple-electrode analysis of primate prefrontal cortex. Journal of Neuroscience 21, 3646-3655.

Constantinidis, C., Williams, G.V., Goldman-Rakic, P.S., 2002. A role for inhibition in shaping the temporal flow of information in prefrontal cortex. Nature Neuroscience 5, $175-180$.

Deadwyler, S.A., Bunn, T., Hampson, R.E., 1996. Hippocampal ensemble activity during spatial delayed-nonmatch-to-sample performance in rats. Journal of Neuroscience 16, 354-372.

Eichenbaum, H., 1993. Thinking about brain cell assemblies. Science 261, 993-994.

Engel, A.K., Fiees, P., Singer, W., 2001. Dynamic predictions: oscillations and synchrony in top-down processing. Nature Review Neuroscience 2, 704-716.

Fee, M., Mitra, P., Kleinfeld, D., 1996. Variability of extracellular spike waveforms of cortical neurons. Journal of Neurophysiology 76, 3823-3833.

Fujisawa, S., Amarasingham, A.., Harrison, M.T., Buzsaki, G., 2008. Behaiviordependent short-term assembly dynamics in the medial prefrontal cortex. Nature Neuroscience 11, 823-833.

Funahashi, S., 2006. Prefrontal cortex and working memory processes. Neuroscience $139,251-261$.

Funahashi, S., Inoue, M., 2000. Neuronal interactions related to working memory processes in the primate prefrontal cortex revealed by cross-correlation analysis. Cerebral Cortex 10, 535-551. 
Fuster, J.M., 2008. The Prefrontal Cortex, fourth ed. New York, Academic Press.

Genovesio, A., Brasted, P. J., Mitz, A.R., Wise, S.P., 2005. Prefrontal cortex activity related to abstract response strategies. Neuron 47, 307-320.

Genovesio, A., Brasted, P.J., Wise, S.P., 2006. Representation of future and previous spatial goals by separate neural populations in prefrontal cortex. Journal of Neuroscience 26,7305-7316.

Gray, C., Maldonado, P., Wilson, M., McNaughton, B., 1995. Tetrodes markedly improve the reliability and yield of multiple single-unit isolation from multi-unit recordings in cat striate cortex. Journal of Neuroscience Methods 63, 43-54.

Gupta, A., Wang, Y., Markram, H., 2000. Organizing principles for a diversity of GABAergic interneurons and synapses in the neocortex. Science 287, 273-278.

Harris, K.D., 2005. Neural signatures of cell assembly organization. Nature Review Neuroscience 6, 399-407.

Harris, K.D., Csicsvari, J., Hirase, H., Dragoi, G., Buzsáki, G., 2003. Organization of cell assemblies in the hippocampus. Nature 424, 552-556.

Hebb, D.O., 1949. The Organization of Behavior-A Neuropsychological Theory. New York, Wiley.

Hempel, C.M., Hartman, K.H., Wang, X.-J., Turrigiano, G.G., Nelson, S.B., 2000. Multiple forms of short-term plasticity at excitatory synapses in rat medial prefrontal cortex. Journal of Neurophysiology 83, 3031-3041.

Henze, D., Borhegyi, Z., Csicsvari, J., Mamiya, A., Harris, K., Buzsaki, G. 2000. Intracellular features predicted by extracellular recordings in the hippocampus in vivo. Journal of Neurophysiology 84, 390-400. 
Ikegaya, Y., Le Bon-Jego, M., Yuste, R. 2005. Large-scale imaging of cortical network activity with calcium indicators. Neuroscience Research 52, 132-138.

Jones, M.W., Wilson, M.A., 2005. Theta rhythms coordinate hippocampal-prefrontal interactions in a spatial memory task. PLoS Biology 3, e402.

Kahana, M.J., 2006. The cognitive correlates of human brain oscillations. Journal of Neurscience 26, 1669-1672.

König, P., Engel, A.K., and Singer, W., 1996. Integrator or coincidence detector? The role of the cortical neuron revisited. Trends in Neuroscience 19, 130-137.

Kucewicz, M.T., Tricklebank, M.D., Robinson E.S.J., Jones, M.W., 2012. Hippocampal-prefrontal cortical neuronal assemblies in operant delayed non-match to position working memory task. 42th Society for Neuroscience Annual Meeting, 918.11/DDD68.

Lewicki, M., 1994. Bayesian modelling and classification of neural signals. Neural Computation 6, 1005-1030.

Lewicki, M., 1998. A review of methods for spike-sorting: the detection and classification of neural action potentials. Network-computation in Neural Systems 9, R53-R78.

Lopes-dos-Santos, V., Conde-Ocazionez, S., Nicolelis, M.L., Ribeiro, S.T., Tort, A.B.L., 2011. Neuronal assembly detection and cell membership specification by principal component analysis. PLoS One 6, e20996.

Markram, H., Toledo-Rodriguez, M., Wang, Y., Gupta, A., Silberberg, G., Wu, C., 2004. Interneurons of the neocortical inhibitory system. Nature Review Neuroscience 5, 793-807. 
McNaughton, B.L., Barnes, C.A., Meltzer, J., Sutherland, R.J., 1989. Hippocampal granule cells are necessary for normal spatial learning but not for spatially-selective pyramidal cell discharge. Experimental Brain Research 76, 485-496.

Miller, E.K., 2000. The prefrontal cortex and cognitive control. Nature Review Neurscience 1, 59-65.

Mongillo, G., Barak, O., Tsodyks, M., 2008. Synaptic theory of working memory. Science 319, 1543-1546.

Nakazono, T., Takahashi, S. and Sakurai, Y., 2012. Multi-neuronal activities in prefrontal cortex and hippocampus during a rule-switching task in rats. 42th Society for Neuroscience Annual Meeting, 706.18/EEE34.

Passingham, R.E., Wise, S.P., 2012. The Neurobiology of the Prefrontal Cortex. Oxford, Oxford University Press.

Peyrache, A., Khamassi, M., Benchenane, K., Wiener, S. I., Battaglia, F.P., 2009. Replay of rule-learning related neural patterns in the prefrontal cortex during sleep. Nature Neuroscience 12, 919-26.

Pipa,G., Munk, M.H., 2011. Higher order spike synchrony in prefrontal cortex during visual memory. Frontiers in Computational Neuroscience 5, doi: 10.3389/fncom.2011.00023.

Riehle, A., Grün, S., Diesmann, M., Aertsen, A., 1997. Spike synchronization and rate modulation differentially involved in motor cortical function. Science 278, 19501953.

Sakamoto, K., Mushiake, H., Saito, N., Aihara, K., Yano, M., Tanji, J., 2008. Discharge synchrony during the transition of behavioral goal representations encoded by discharge rates of prefrontal neurons. Cerebral Cortex 18, 2036-45. 
Sakurai, Y., 1996a. Hippocampal and neocortical cell assemblies encode memory processes for different types of stimuli in the rat. Journal of Neuroscience 16, 28092819.

Sakurai, Y., 1996b. Population coding by cell assemblies-what it really is in the brain. Neuroscience Research 26, 1-16.

Sakurai, Y., 1999. How do cell assemblies encode information in the brain? Neuroscience \& Biobehavioral Review 23, 785-796.

Sakurai, Y., 2002. Coding of temporal information by hippocampal individual cells and cell assemblies in the rat. Neuroscience $115,1153-1163$.

Sakurai, Y., Takahashi, S., 2006. Dynamic synchrony of firing in the monkey prefrontal cortex during working memory tasks. Journal of Neuroscience 26, 10141-10153.

Sakurai, Y., Takahashi, S., 2008. Dynamic synchrony of local cell assembly. Reviews in Neuroscience 19, 425-440.

Sakurai, Y., Takahashi, S., Inoue, M., 2004. Stimulus duration in working memory is represented by neuronal activity in the monkey prefrontal cortex. European Journal of Neuroscience 20, 1069-1080.

Seidemann, E., Meilijson, I., Abeles, M., Bergman, H., Vaadia, E., 1996. Simultaneously recorded single units in the frontal cortex go through a sequence of discrete and stable states in monkeys performing a delayed localization task. Journal of Neuroscience $16,752-768$.

Sussilo, D., Toyoizumi, T., Maass, W., 2006. Self-tuning of neural circuits through short-term synaptic plasticity. Journal of Neurophysiology 97, 4079-4095. 
Takahashi, S., Anzai, Y., Sakurai, Y., 2003a. Automatic sorting for multi-neuronal activity recorded with tetrodes in the presence of overlapping spikes. Journal of Neurophysiology $89,2245-2258$.

Takahashi, S., Anzai, Y., Sakurai, Y., 2003b. New approach to spike sorting for multineuronal activities recorded with a tetrode - how ICA can be practical. Neuroscience Research 46, 265-272.

Tsujimoto, S., Genovesio, A., Wise, S.P., 2008. Transient neuronal correlations underlying goal selection and maintenance in prefrontal cortex. Cerebral Cortex 18, $2748-2761$.

Vaadia, E., Haalman, I., Abeles, M., Bergman, H., Prut, Y., Slovin, H., Aertsen, A., 1995. Dynamics of neuronal interactions in monkey cortex in relation to behavioural events. Nature 373, 515-518.

Wang, Y., Markram, H., Goodman, P.H., Berger, T.K., Ma, J., Goldman-Rakic, P.S., 2006. Heterogeneity in the pyramidal network of the medial prefrontal cortex. Nature Neuroscience 9, 534-542.

Watanabe, M., 2007. Role of anticipated reward in cognitive behavioral control. Current Opinion in Neurobiology 17, 213-219.

Wilson, M.A., McNaughton, B.L., 1993. Dynamics of the hippocampal ensemble code for space. Science 261, 1055-1058. 


\section{Figure legends}

Fig. 1. A, An example of difference correlograms from a pair of neurons showing taskdependent synchrony. The correlograms were constructed for the 3 event periods (sample stimulus, retention interval, and comparison stimulus) during the 2 tasks [delayed match-to-sample of duration (DMS-D) and delayed match-to-sample of color (DMS-C)]. Each correlogram represents the number of spikes per bin that occurred in one neuron (target neuron) before and after spikes in another neuron (reference neuron). The bin width is $1 \mathrm{~ms}$. The horizontal values indicate time in ms between -100 and +100 , and the ordinate values are accumulated numbers of spikes per bin. A band of $99.5 \%$ confidence limits is shown by broken lines. Red correlograms mean that they have significant synchrony, which was defined as those with more than 1 bin over 1.5 times the band between the upper and lower confidence limits in each correlogram. The rightupper portion of each correlogram shows the total number of spikes that were obtained from the reference neuron (R) and target neuron $(\mathrm{T})$ of the pair to calculate the correlograms. The bottom of the figure shows the data ID. The neurons of this pair were recorded from 1 tetrode. B, Difference correlograms presented at a higher temporal resolution in order to show the detailed structures of the peaks. The bin width is $0.1 \mathrm{~ms}$, and the range of the horizontal values is -10 and $+10 \mathrm{~ms}$. C, An example of scatter plots of spike clusters from the 2 neurons of the pair. The $x$ - and $y$-axes represent the peak amplitude of the spikes detected by ch. 2 and the valley amplitude of the spikes detected by ch. 4 , respectively, of the 4 chs of the tetrode. Green and blue points represent the reference (R) and target (T) neurons, respectively. A total of 28 types of scatter plots of spike clusters (peak amplitude $\times$ valley amplitude $\times 4$ chs.) were made for each neuron, 
and this is one example that shows the separated clusters. D, Averaged waveforms of the 2 neurons that were recorded from each ch. of the tetrode across all trials of the 2 tasks. The green and blue waveforms represent the reference $(\mathrm{R})$ and target $(\mathrm{T})$ neurons, respectively. (From Sakurai and Takahashi, 2006, with permission)

Fig. 2. Time-resolved contrast of the rates of joint-spike events (JSE) with complexities (y-axis) ranging from 2 to 8 as a function of time with respect to the onset of sample stimuli ( $x$-axis). Z-scores (color scale) were computed by taking the difference between the rates of JSEs in the correct and incorrect trials divided by the standard deviation of the JSE rates obtained in permuted trials with correct and erroneous behavioral responses. In the color scale, green and red indicate that spike synchrony was significantly increased in correct trials and in error trials, respectively. (From Pipa and Munk, 2011, with permission)

Fig. 3. Population-averaged coincidence histograms, which were calculated from neuron pairs with significant positive correlations. Background shading shows the standard error of the means. The arrow marks a period after the goal had been chosen, but feedback had not yet arrived. Note that the plot is divided into 2 parts, with one aligned on cue onset and the other on cue offset, because the duration of the cue was variable and unpredictable. (From Tsujimoto et al., 2008, with permission)

Fig. 4. A, Firing patterns of neurons recorded simultaneously in medial prefrontal cortex (mPFC). Each row represents the position-dependent firing rate of a single neuron (normalized relative to its peak firing rate). Neurons were ordered by the 
location of their peak firing rates relative to the rat's position in the maze. Top frames, neurons with higher peak rates during left-turn trials; Bottom frames, higher peak rates during right-turn trials. Third columns, segments with significantly higher discharge rates during left (blue) or right (red) turns. B, Short-term cross-correlograms between a putative pyramidal cell (cell 1) and interneuron (cell 2) as a function of the rat's position in 40 sliding subsegments of the maze (each cross-correlogram window overlapped by 4 segments) during left-turn trajectories. The 0.5 point indicates a turning point where the rats chose either the right or the left arm. Top right, a mean cross-correlogram in the session. Top left, superimposed traces of the mean waveforms (black) and single spikes (gray) of the respective units $(1 \mathrm{~Hz}-8 \mathrm{kHz})$. Cells 1 and 2 were recorded by different shanks. (From Fujisawa et al., 2008, with permission)

Fig. 5. Time course of functional connectivity (FC) changes across training sessions. A, B, Box plots showing the distribution of changes of FC ( $\Delta \mathrm{FC})$ of neuron pairs that were recorded across multiple days in phases I and II. Positive-negative numbers on the ordinate denote enhancement-reduction in FC across 2 days. The numbers on the abscissas of the graphs that are shown in A-F denote the time period of the sessions. The diagram shows the median values (horizontal lines inside boxes), interquartile distances, and upper and lower $10 \%$ values. C, D, The time course of $\Delta \mathrm{FC}$ variances (Var) in each training phase. $\Delta \mathrm{FC}$ was computed based on the neural activity during the baseline period before each session (wherein animals sat quietly on a pedestal in the recording room for $10 \mathrm{~min}$ before the task). E, F, $\Delta \mathrm{FC}$ was computed based on the neural activity during task-1 performance. The solid lines were determined by linear regression. 
Fig. 6. The procedure of the automatic sorting of multineuronal activities with the combination of ICA and k-means clustering. ICA, Independent component analysis. IC, Independent component. (From Takahashi, et al., 2003b, with permission)

Fig. 7. A detailed example of raw signals from a single tetrode at slow (A) and fast (B) time scales. The box of dotted lines (B) is a period when spikes from 2 of the single cells that were included in the tetrode data overlap. C, Overlapped and separated waveforms for the overlapping spikes. (From Sakurai and Takahashi, 2006, with permission).

Fig. 8. An example of the raw data of a single neuron that was separated by the present method from the tetrode data shown in Fig. 8. The neuronal activity was recorded for 2 h during the behavioral tasks. A, Scatter plot of the spike cluster. Each black point in the scatter plot represents a spike. The $\mathrm{x}$ - and $\mathrm{y}$-axes represent valley amplitudes of the spikes detected by chs. 1 and 4, respectively, of the 4 tetrode chs. B, Averaged waveforms recorded from each ch. of the tetrode at the 2 plots of spikes ( $\mathrm{a}$ and $\mathrm{b}$ ). $\mathbf{C}$, Auto-correlogram of the firing of the single neuron. Bin width is $0.5 \mathrm{~ms}$. The horizontal values indicate time in $\mathrm{ms}$, and the ordinate values are the accumulated numbers of spikes per bin. (From Sakurai and Takahashi, 2006, with permission)

Fig. 9. Proportions of the neuronal pairs showing fixed synchrony, task-dependent synchrony, and event-task-dependent synchrony that were recorded from 1 tetrode (A) and 2 tetrodes (B). Blank bar grams, proportions of the neuronal pairs showing sharp 
synchrony. Dark bar grams, proportions of the neuronal pairs showing broad synchrony. The values in parentheses are the numbers of neuronal pairs (the denominators are the total neuronal pairs showing each synchrony). An asterisk above the bar gram indicates a significantly different proportion between the types of synchrony. (From Sakurai and Takahashi, 2006, with permission) 
A

DMS-D
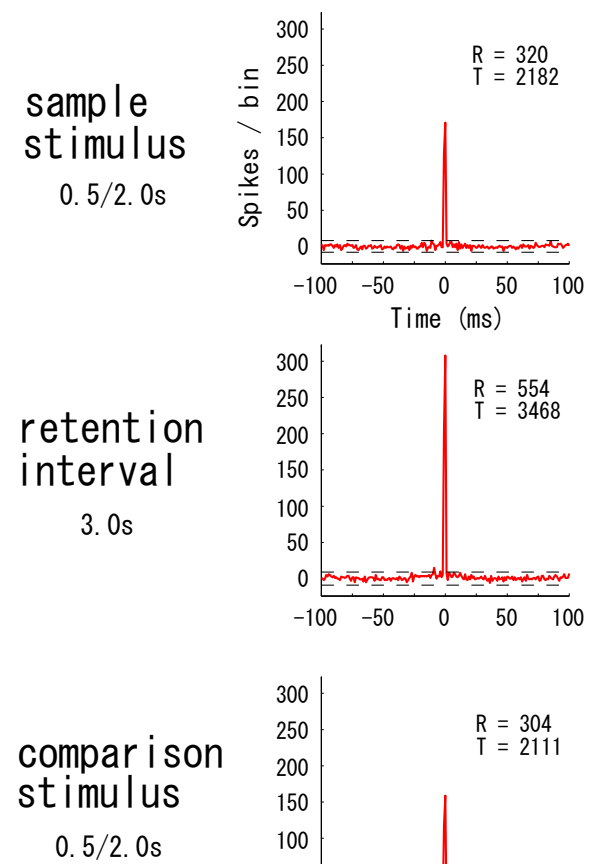

$0.5 / 2.0 \mathrm{~s}$

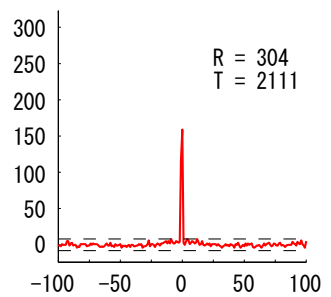

s330e456d4-7a vs. $s 330$ e456d4-5a
B
DMS-D
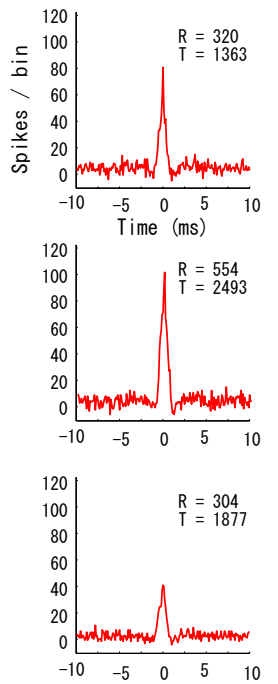

DMS-C
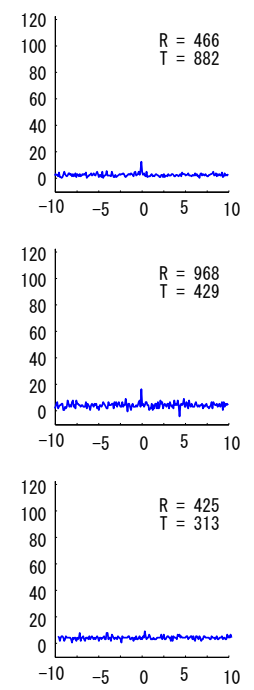

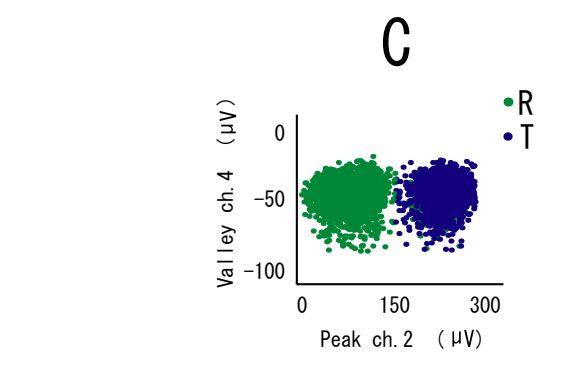

D

$\mathrm{R} \quad$ DMS-D T

ch. 1 ch. 2 ch. 3 ch. 4

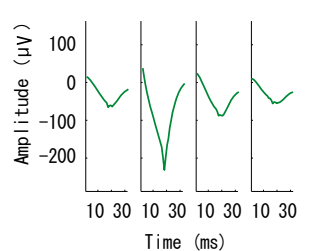

ch. 1 ch. 2 ch. 3 ch. 4

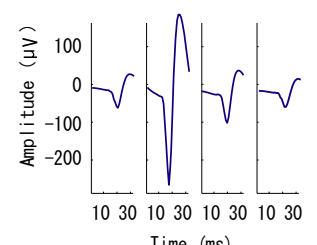

$\mathrm{R}=425$
$\mathrm{~T}=2413$

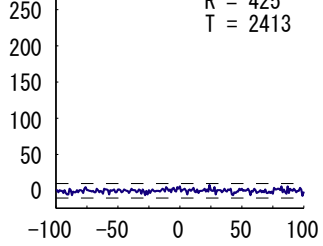

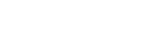

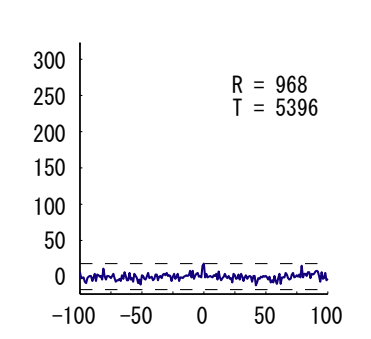

D

Fig. 1 (Sakurai et al.) 


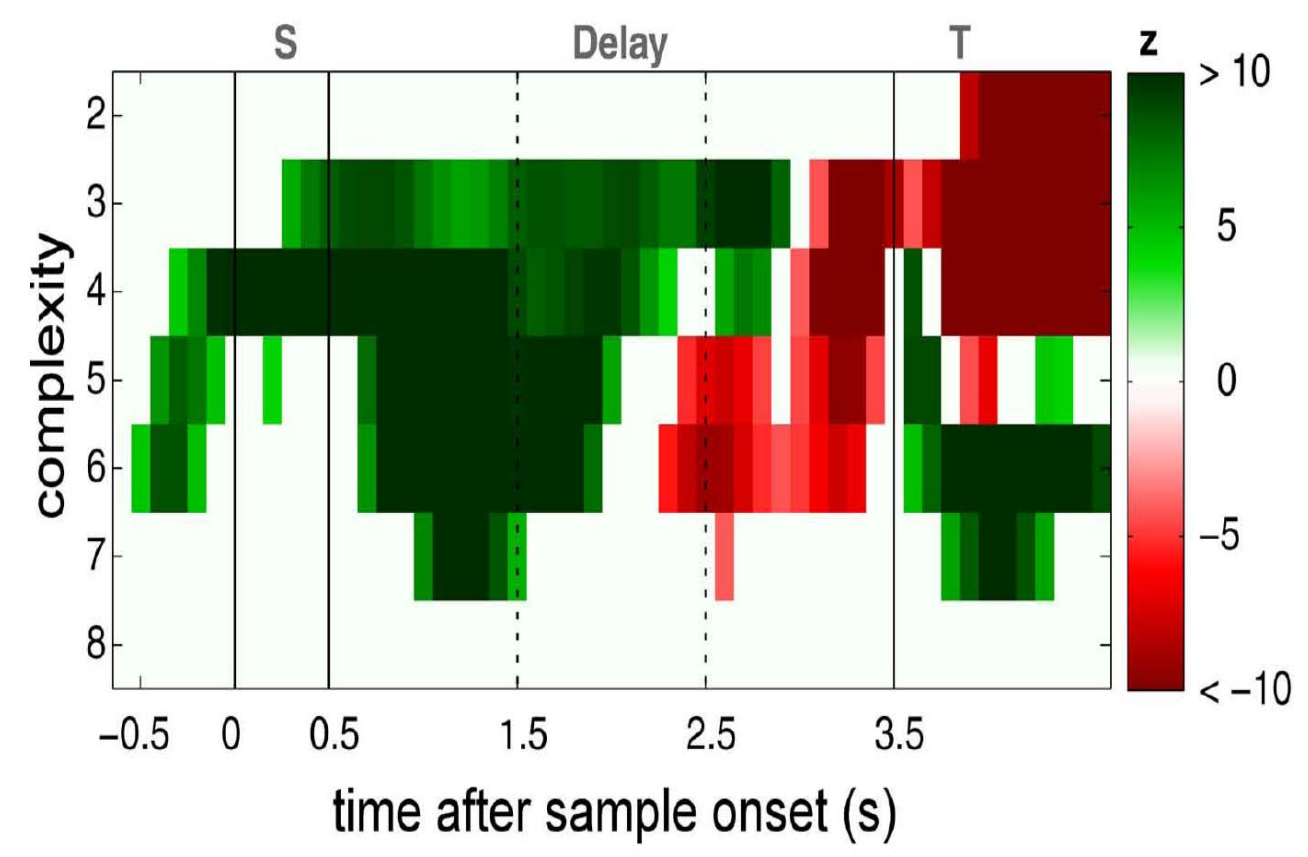

Fig.2 (Sakurai et al.) 


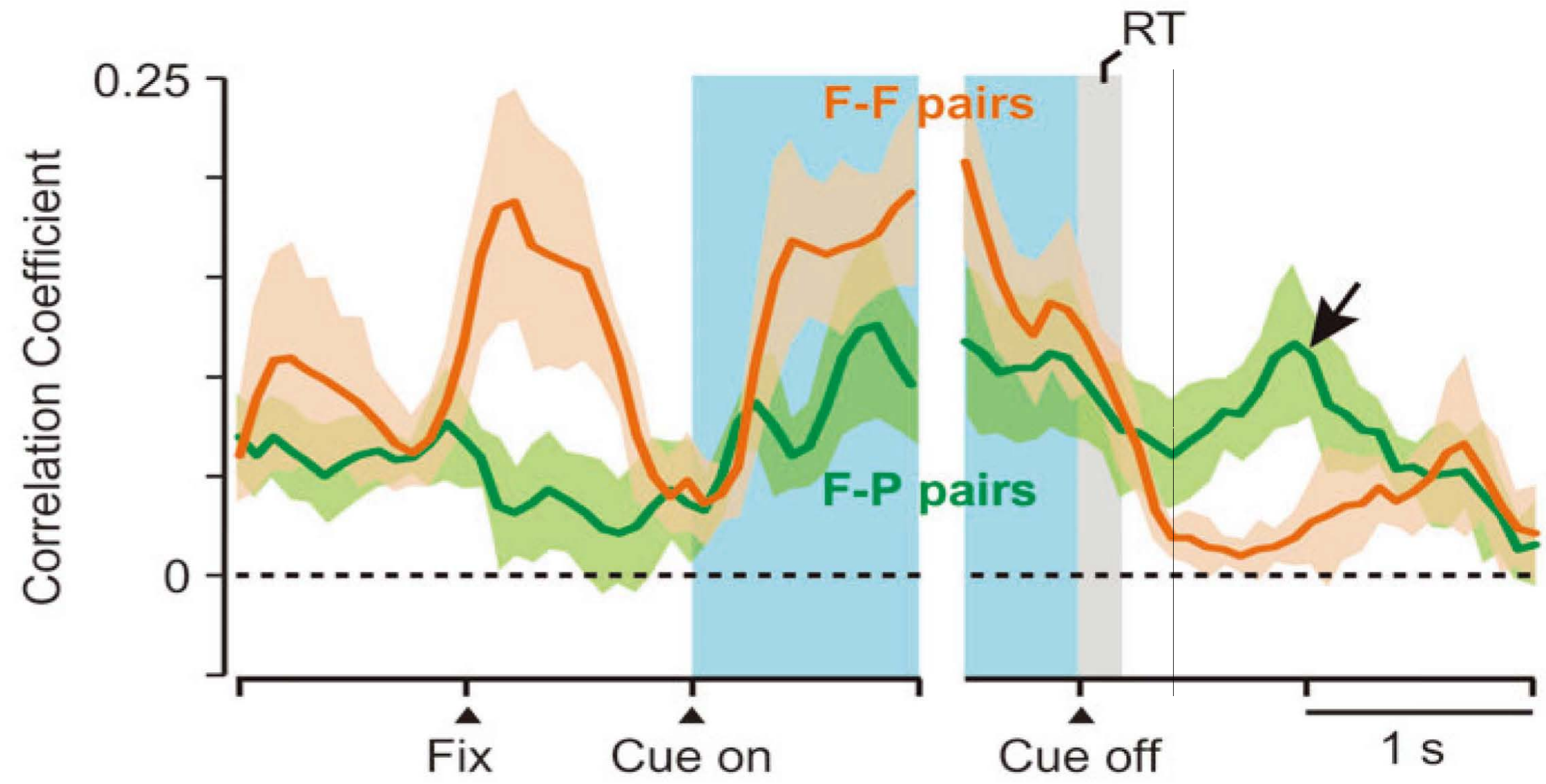

Fig.3 (Sakurai et al.) 
A

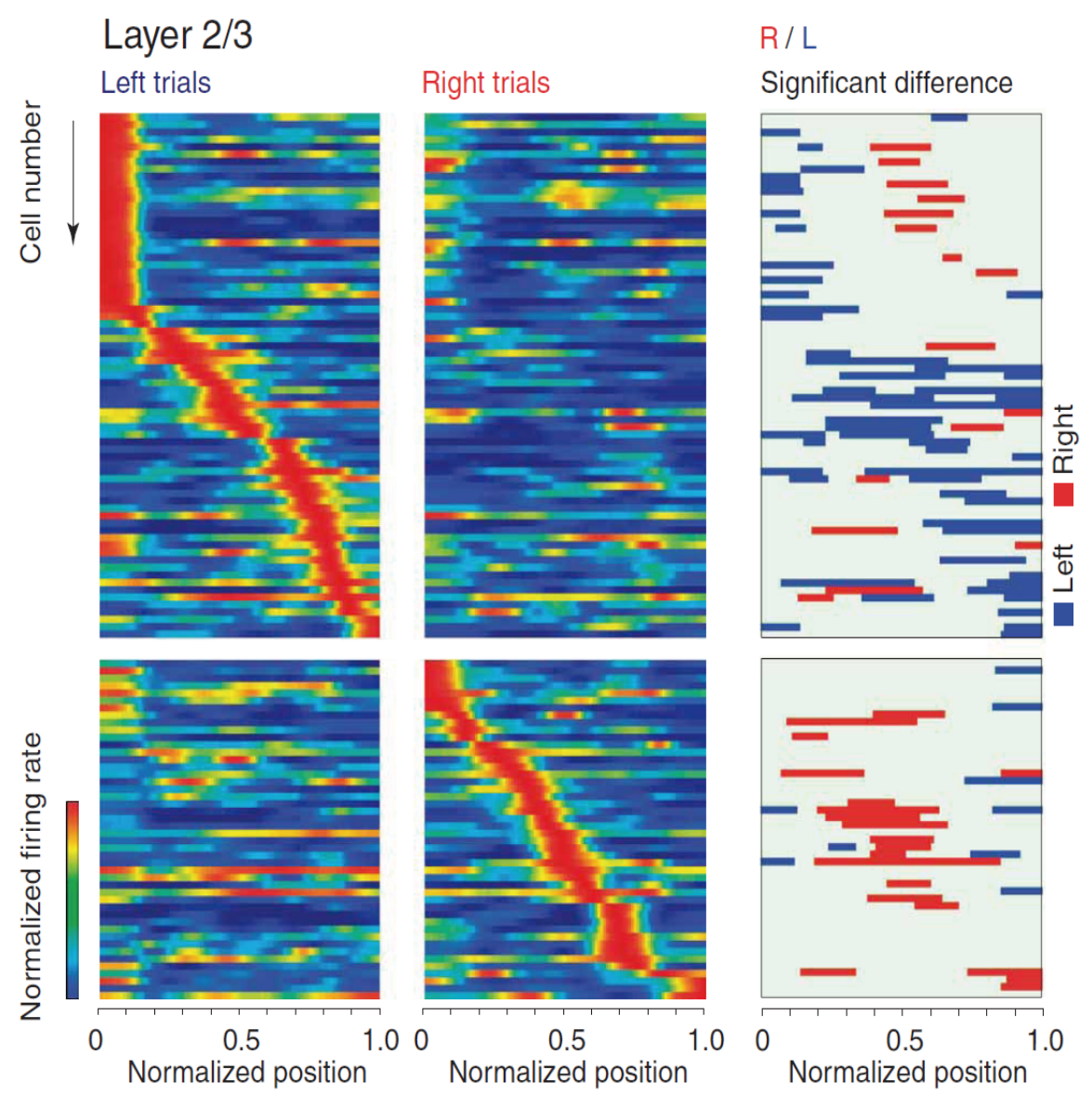

B

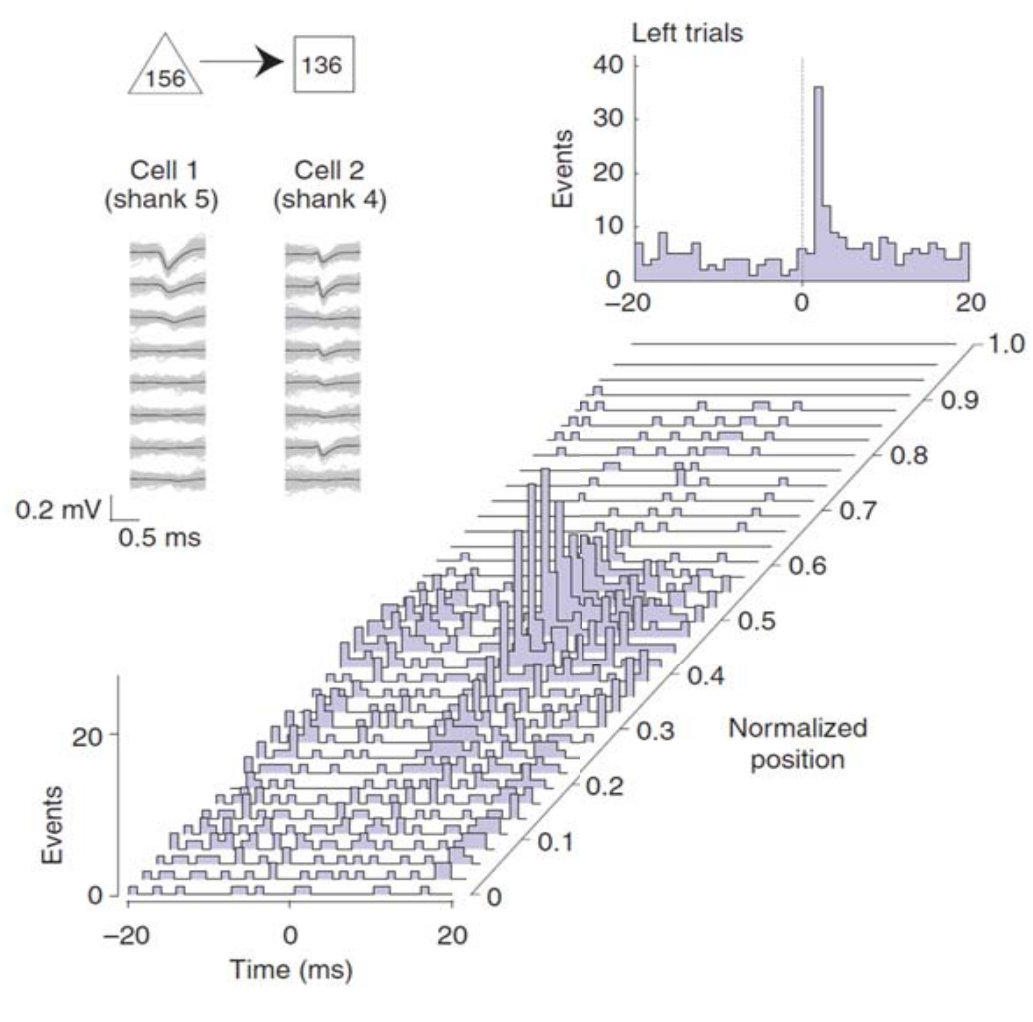

Fig.4 (Sakurai et al.) 

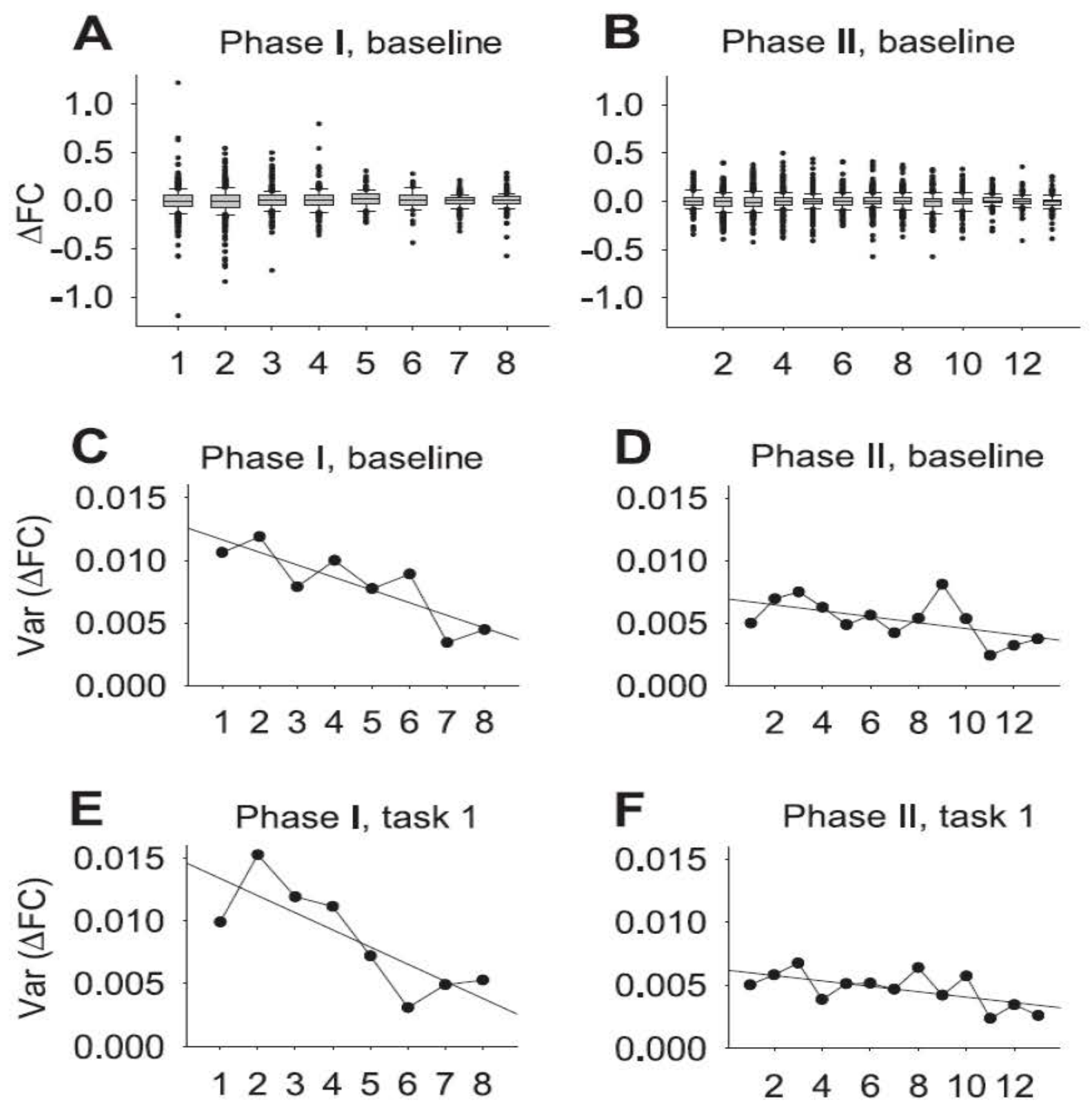

Fig.5 


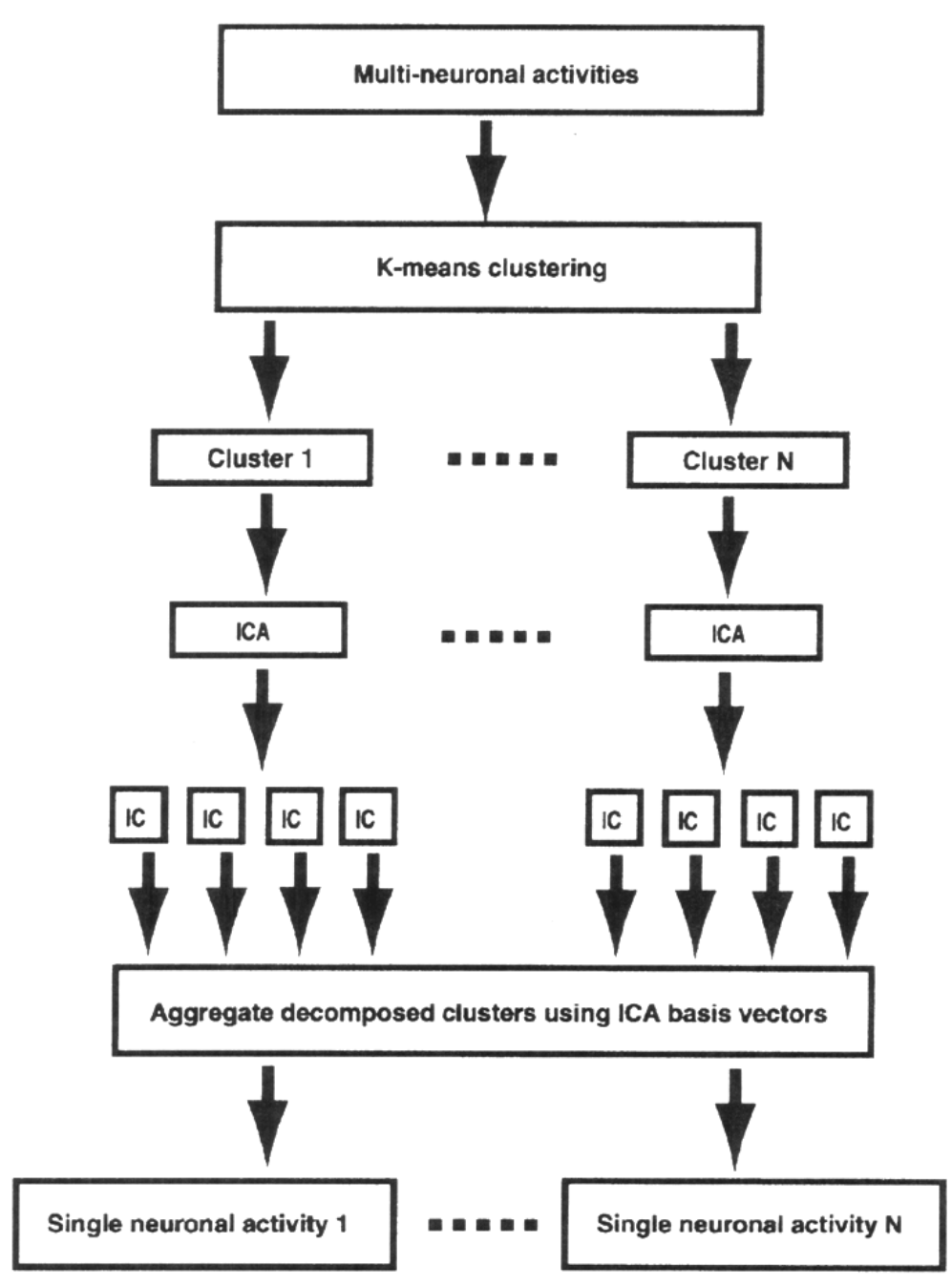

Fig.6 
ch. 1

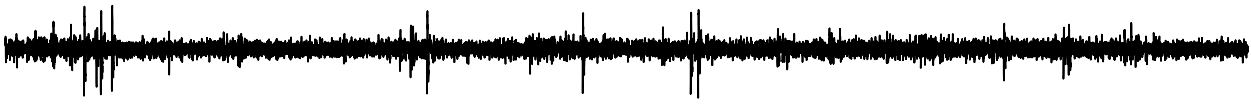

ch. 2

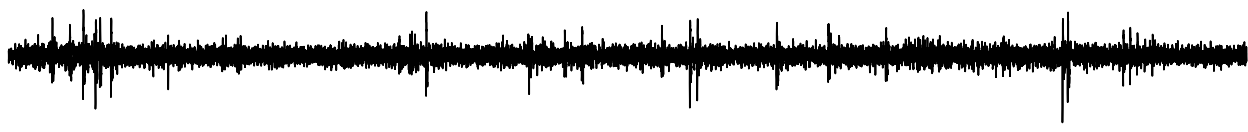

A

ch. 3

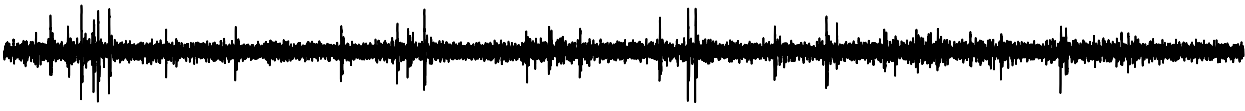

ch. 4

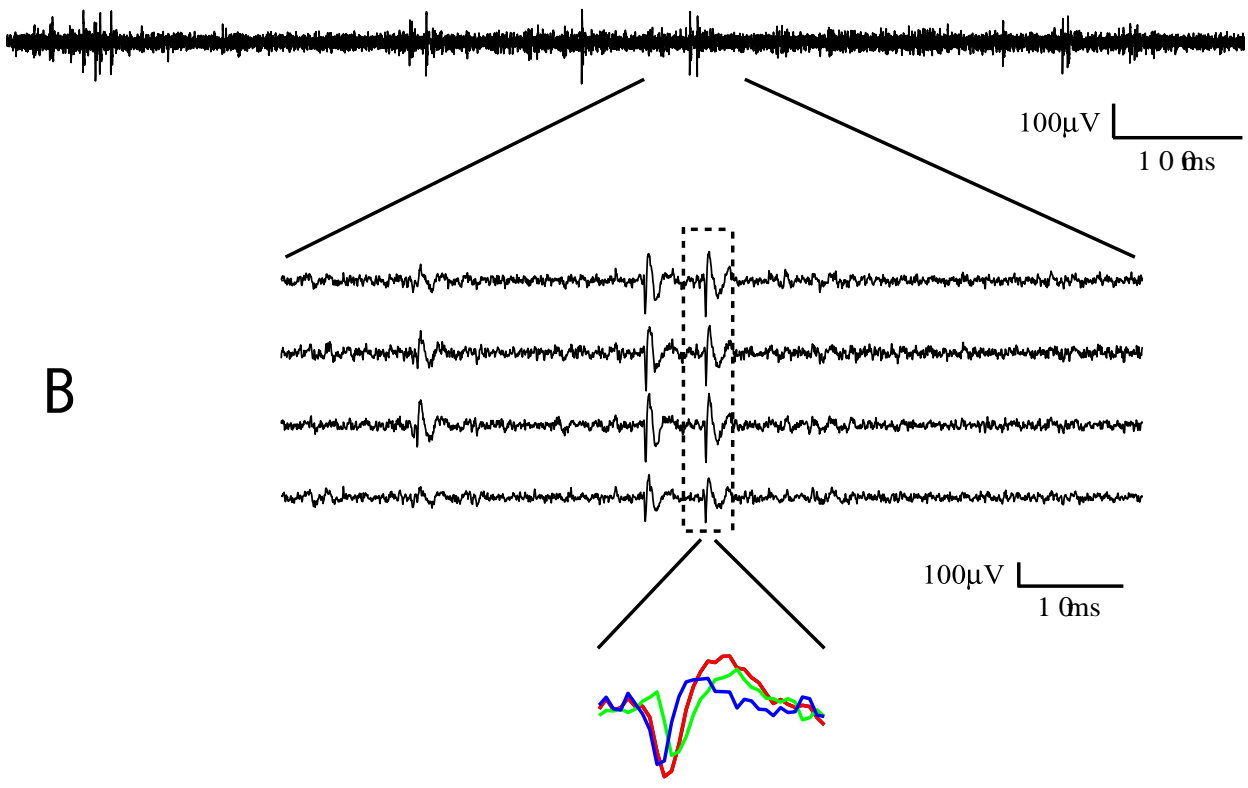

C
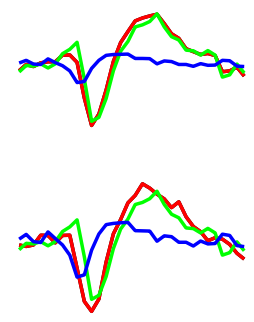

_ Over lapped waveform

— Neuron 1 waveform

- Neuron 2 waveform

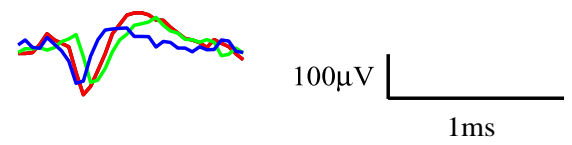

DATA S450E15D5 


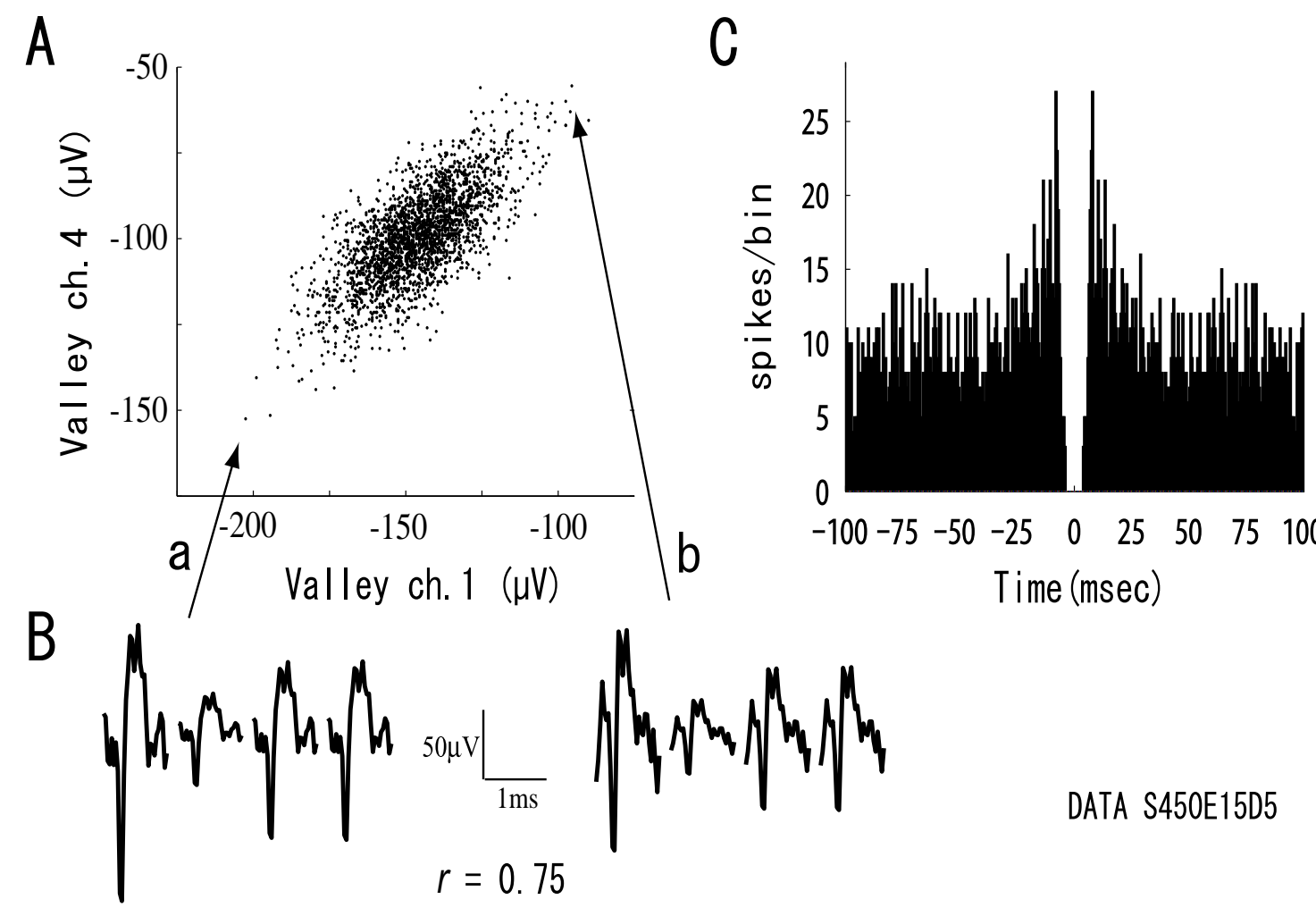

ch. 1 ch. 2 ch. 3 ch. $4 \quad$ ch. 1 ch. 2 ch. 3 ch. 4

Fig.8 


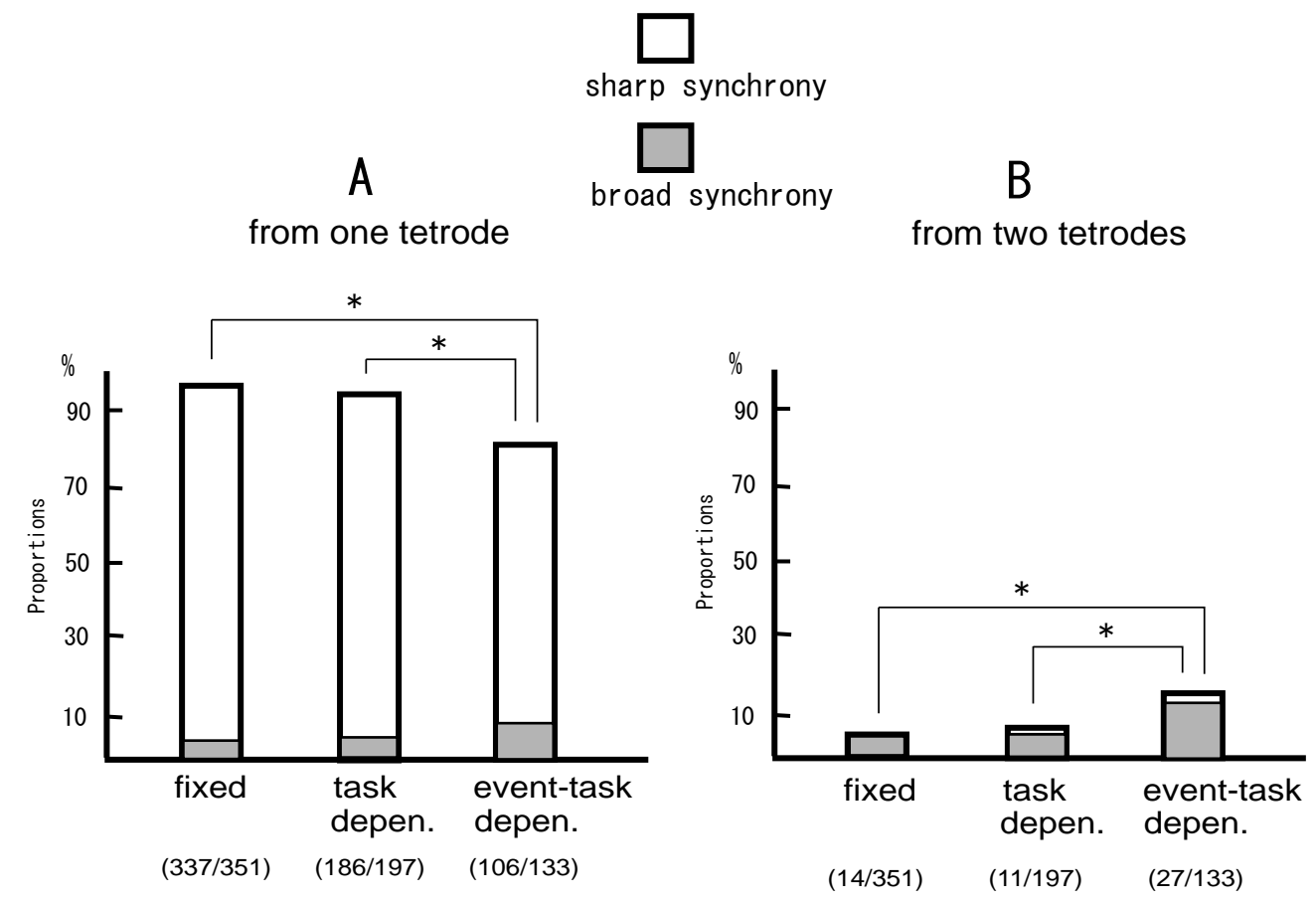

Fig.9 